\title{
Improved Thévenin equivalent methods for real-time voltage stability assessment
}

\author{
Angel Perez * Hjörtur Jóhannsson* Jacob Østergaard* Mevludin Glavic ${ }^{\dagger} \quad$ Thierry Van Cutsem ${ }^{\ddagger}$ \\ * Technical University of Denmark \\ $\dagger$ Dept. of Electrical Engineering and Computer Science, University of Liège, Belgium \\ $\ddagger$ Fund for Scientific Research (FNRS) at the Dept. Elec. Eng. and Comp. Science, University of Liège, Belgium
}

\begin{abstract}
An improved Thévenin equivalent method for realtime voltage stability assessment that uses wide-area information from synchrophasors is proposed. The improvements are a better modeling of the limited synchronous generators, and a processing that anticipates the effect of field current limiters, before the latter are activated. Several study cases using detailed dynamic simulations of the Nordic test system have been used to assess the performance of the proposed improvements. Their effectiveness is analyzed and, based on the results, their possible application in combination with the sensitivity-based voltage stability assessment method is explored.

Index Terms-Wide-area monitoring, voltage stability, realtime instability detection, Thévenin equivalent, stability limit, overexcitation limiters, sensitivity analysis.
\end{abstract}

\section{INTRODUCTION}

The applications of synchrophasors and the number of installed units has increased steadily $[1,2]$ and their potentials deserve being further explored. Initiatives like $\mathrm{NASPI}^{1}$ and $\mathrm{SOSPO}^{2}$ [3] are examples of these efforts.

This work is part of the SOSPO project, whose objective is to provide a set of fast stability assessment methods for a power system envisioned to have a high share of wind energy and other fluctuating energy sources [4-7]. In this scenario, it is foreseen that real-time applications will benefit from the availability synchrophasors technology to cope with the new challenges.

In the specific case of voltage stability [8,9], the Thévenin Equivalent Methods (TEMs) and the sensitivity-based method [10] were identified in [11] as suitable for wide-area real-time monitoring from a control center. Both approaches have low computational complexity, making them suitable for fast realtime applications. For a review of existing methods, refer to $[11,12]$.

Some limitations of the TEMs were identified in [11], after which a number of improvements have been explored, dealing with: synchronous generator limits [13], wind-farms limits [14] and HVDC interconnections [15]. A further set of improvements is presented in this paper, inspired of techniques

This work has received funding from the Danish Council for Strategic Research as a part of the project Secure Operation of Sustainable Power Systems (SOSPO)

${ }^{1}$ www.naspi.org

${ }^{2}$ www.sospo.dk proposed in [16] in complement to the sensitivity computation and monitoring.

The Nordic system, as described in [17] and with the study cases detailed in $[16,18]$, was selected to validate and assess the modified Thévenin equivalent method proposed in this paper.

Based on the simulation results, synergies between the TEM and the sensitivity method have been explored in order to improve the overall stability assessment, assuming that both approaches are to be applied in parallel.

The paper is organized as follows. The TEM is described and its specific improvements are detailed in Section II. The required pre-processing of the measurements obtained from Phasor Measurement Units (PMUs) is also briefly discussed. The set of study cases used to benchmark the method is described in Section III. Based on the simulation results, the methods capabilities are discussed and an initial exploration of the synergies between the TEM and the sensitivity methods is presented in Section IV. Some concluding remarks are offered in Section V.

\section{IMPROVED THÉVENIN EQUIVALENT METHOD}

The proposed TEM method is an extension of the one based on $[13,14]$. The steps are shown in Fig. 1. The main modifications considered in this paper deal with the following:

- anticipation of the OverExcitation Limiter (OEL) activation, using the technique described in [10]. Namely, as soon as a generator field current exceeds the limit, and before it is effectively limited by the OEL, the equations of the limited generator are anticipatively used in the Thévenin equivalent computation;

- improved calculation of the generator e.m.f. The latter is used as detailed in [13]. The saturated q-axis e.m.f. is considered, as described in [10].

\section{A. Description of modified Thévenin equivalent method}

The TEM computes the Thévenin equivalent seen from each bus, as shown in Fig. 2. The first descriptions of TEM used a least-square procedure to determined the parameters from local measurements [19]. The approach followed in this paper is to compute the parameters from wide-area measurements together with the network model $[13,14,20]$. 


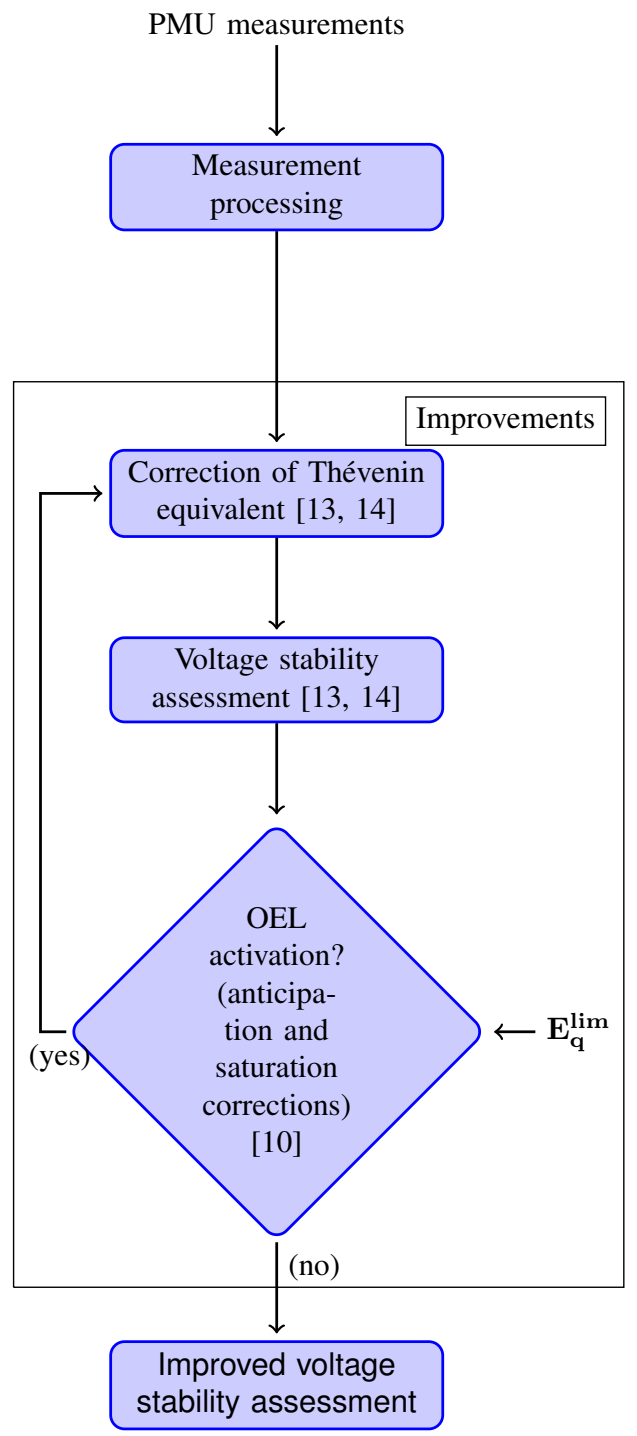

Figure 1. Proposed TEM method improvements

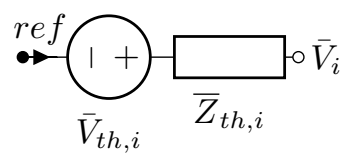

Figure 2. Thévenin equivalent circuit computed at bus $i . \bar{V}_{t h, i}$ and $\bar{Z}_{t h, i}$ are the Thévenin voltage and impedance, respectively

The Thévenin e.m.f. is computed as a linear combination of the individual generator e.m.f.'s according to $[4,13,14]$ :

$$
\bar{V}_{t h, i}=\sum_{k \in G} \overline{G T C}_{i, k} \bar{E}_{g, k}
$$

where $\bar{V}_{t h, i}$ is the Thévenin voltage at bus $i, G$ is the set of buses that have an attached generator, $\bar{E}_{g, k}$ is the estimated e.m.f. of generator $k$, and GTC stands for Generator Transformation Coefficients.

As already mentioned, the main improvement to the original
TEM is the capability to correct the individual generator e.m.f.'s to take into account the limits. Assuming that the $k$-th generator is itself represented by an e.m.f. $\bar{E}_{g, k}$ behind an impedance $\bar{Z}_{g, k}=j X_{g, k}$, where the armature resistance is neglected [10], the following equation can be written:

$$
\bar{E}_{g, k}=\bar{V}_{k}+j X_{g, k} \bar{I}_{g, k}
$$

where $\bar{V}_{k}$ is the generator terminal voltage, and $\bar{I}_{g, k}$ its current. Both of them are obtained from synchrophasor measurements.

In order to account for an OEL activation, $\bar{E}_{g, k}$ and $\bar{Z}_{g, k}$ have to be related to the model of the synchronous machine under limit and with saturation effects [10]. Dropping the index $k$ for simplicity, this model can be written in $(d, q)$ axes as:

$$
\left[\begin{array}{c}
0 \\
E_{q}^{s}
\end{array}\right]=\left[\begin{array}{c}
V_{d} \\
V_{q}
\end{array}\right]+\left[\begin{array}{cc}
0 & X_{q}^{s} \\
-X_{d}^{s} & 0
\end{array}\right]\left[\begin{array}{c}
I_{d} \\
I_{q}
\end{array}\right]
$$

where $E_{q}^{s}$ is the generator saturated e.m.f. in the $q$ axis, $X_{q}^{s}$ (resp. $X_{d}^{s}$ ) is the $q$-axis (resp. $d$-axis) synchronous reactance corrected to include saturation as detailed in [10], $V_{q}$ (resp. $V_{d}$ ) is the $q$-axis (resp. $d$-axis) component of the voltage phasor, and $I_{q}$ (resp. $I_{d}$ ) is the $q$-axis (resp. $d$-axis) component of the current phasor.

The link between (2) and (3) can be made by projecting equation (2) on the $d$ and $q$ axes, respectively, while taking into account that $\bar{E}_{g}$ is directed along the $q$ axis. This yields:

$$
\left[\begin{array}{c}
0 \\
E_{g}
\end{array}\right]=\left[\begin{array}{c}
V_{d} \\
V_{q}
\end{array}\right]+\left[\begin{array}{cc}
0 & X_{g} \\
-X_{g} & 0
\end{array}\right]\left[\begin{array}{c}
I_{d} \\
I_{q}
\end{array}\right]
$$

Considering that (3) and (4) are two equivalent representations yields, in the $d$ axis:

$$
X_{g}=X_{q}^{s}
$$

and in the $q$ axis:

$$
E_{g}=E_{q}^{s}+\left(X_{d}^{s}-X_{q}^{s}\right) I_{d}
$$

The anticipative representation of a machine under field current limit is based on the consideration that the unsaturated e.m.f. $E_{q}$, which is proportional to the field current, exceeds the corresponding limit:

$$
E_{q}=k E_{q}^{s}>E_{q}^{l i m}
$$

where $k$ is the saturation coefficient and $E_{q}^{\lim }$ is the value of $E_{q}$ under the field current limit, as defined in [10]. When the inequality (7) holds true, the machine is switched under limit and is represented by an e.m.f. $\bar{E}_{g}^{\text {lim }}$ of constant magnitude behind the saturated reactance $X_{q}^{s}$. These are updated with each new PMU snapshot.

Denoting by $G_{v c}$ (resp. $G_{l i m}$ ) the set of generators under voltage control (resp. under field current limit), the Thévenin voltage is obtained from Eq. (1) by separating the contributions of both types of generators:

$$
\bar{V}_{t h, i}=\sum_{k \in G_{v c}} \overline{G T C}_{i, k} \bar{E}_{g, k}+\sum_{k \in G_{l i m}} \overline{G T C}_{i, k} \bar{E}_{g, k}^{l i m}
$$

The value of $\bar{E}_{g, k}$ when the generator is under voltage control $\left(k \in G_{v c}\right)$ is represented as in [13], with a phasor 
that equals to the one where the voltage is being controlled and $\bar{Z}_{g, k}=0$; it is updated with each new PMU snapshot.

Using the so estimated $\bar{V}_{t h, i}$ value, the maximum power that can be extracted from the Thévenin equivalent at bus $i$ is computed, taking anticipatively into account the OEL activation. The so obtained maximum power will be denoted by $S_{\max m}$. It will be compared with the maximum power obtained from the original TEM (i.e. without OEL anticipation, nor saturation effects), which will be denoted by $S_{\max } t h$.

\section{B. Processing of synchrophasor measurements}

The proposed TEM is applied using the following real-time data and making the following assumptions:

- enough PMUs are installed to provide full observability of the system. Although these measurements should be processed by a state estimator, this step is not considered here;

- only a moving average filter is applied to the measurements, as described in [16]. This filter uses a window of 20 samples;

- the sampling rate of PMU measurements is $50 \mathrm{~Hz}$;

- synchronized voltage and current phasors are synthesized from detailed time-domain simulations of the system, using the RAMSES software developed at the University of Liège [21]. The uncertainty in the measurements has been considered using the methodology in [20];

- since the evaluation of long-term voltage stability is of interest, the assessment has been started a few (3 to 15) seconds after the disturbance has been applied to the model.

\section{Detection of an impending instability}

The detection of an impending instability is obtained by comparing the load apparent $S$, active $P$ and reactive power $Q$ against the maximum power that is computed from the Thévenin equivalent. The boundary is crossed when any of the $P, Q, S$ values of the load power is greater than the maximum power.

The above comparison is sketched in Fig. 3, which also suggests that it is possible to obtain a security margin from the difference between the maximum and the load powers. The continuous monitoring of this margin offers the possibility of raising an early alarm.

Furthermore, the weakest buses are identified as those where the above margin is the smallest.

Let us recall that in the improved TEM proposed in this paper, the activation of OELs is anticipated, assuming the activation of OELs as soon as the field current exceeds the limit. The actual critical point (or stability boundary) [16] will be crossed when the OELs are effectively activated.

\section{Simulation RESUlTS}

\section{A. Test System}

The Nordic system, as described in [10, 18] and shown in Fig. 4 was used. As in [16], bus 1041 was identified as the critical bus (i.e. the bus where the TEM-based alarm is

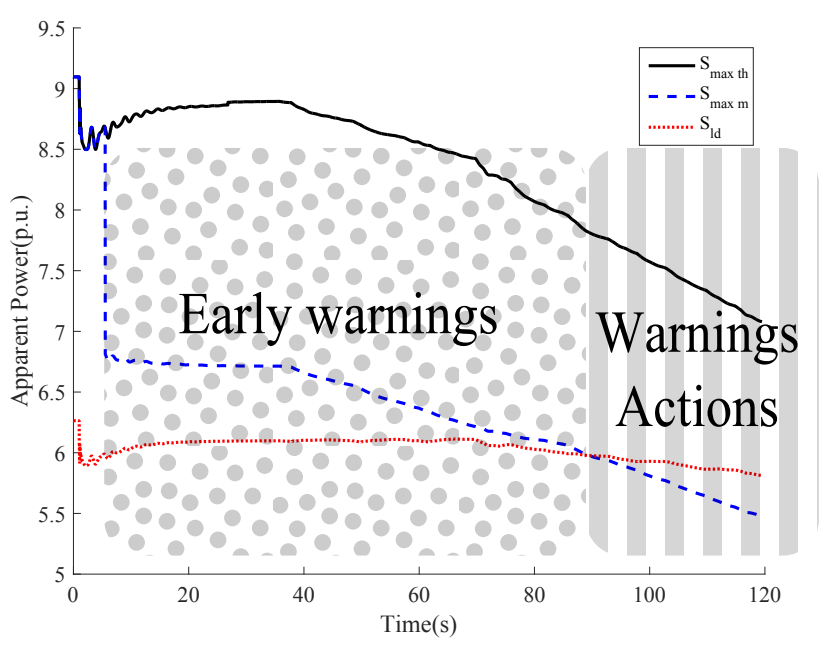

Figure 3. Time evolution of maximum and load powers in an unstable scenario, depicting the regions for early warning (polka dots) and definitive warnings and actions (stripes)

raised first) and the TEM indices shown in this section were computed at this bus.

The following two cases were considered.

1) Case A: unstable scenario. As defined in [16], a threephase fault on the line 4032-4044 (near bus 4044) occurs at $t=1 \mathrm{~s}$. It is cleared at $t=1.1 \mathrm{~s}$ by opening the faulted line.

2) Case B: stable scenario. Case A has been stabilized by automatic emergency control in the form of undervoltage load shedding at buses 1041, 1043 and 1045. Load shedding is actuated in several steps, starting at $t=116 \mathrm{~s}$.

\section{B. Results: Case A}

The system response in Case A is given in Fig. 5, showing the progressive fall of the voltage at bus 1041 under the effect of load tap changers controlling distribution voltages as well as OELs acting on several generators successively. Compared to the response given in [17], some fast transients have been filtered by the moving average filter mentioned in Section II.B.

The voltage stability indices obtained by the original as well as the modified TEM are shown in Fig. 6. The improved TEM identified the impending instability (owing to $S_{l d}>S_{\max m}$ ) before the original TEM $\left(S_{l d}>S_{\max t h}\right)$. The progressive variation of the indices is easily seen in the figure. It offers operators and algorithms an early warning of the developing instability.

\section{Results: Case B}

The stabilized system response in Case B is shown in Fig. 7, where the voltage evolution at bus 1041 is compared with the one in Case A. Figure 8 shows the corresponding indices. An early warning is produced by the improved TEM $\left(S_{l d}>\right.$ $S_{\max m}$ ) before load shedding takes place.

After load shedding and the anticipated limit has been crossed, the power of the load becomes lower than the maximum $\left(S_{\max m}\right)$ and, therefore, the case is regarded as stable 


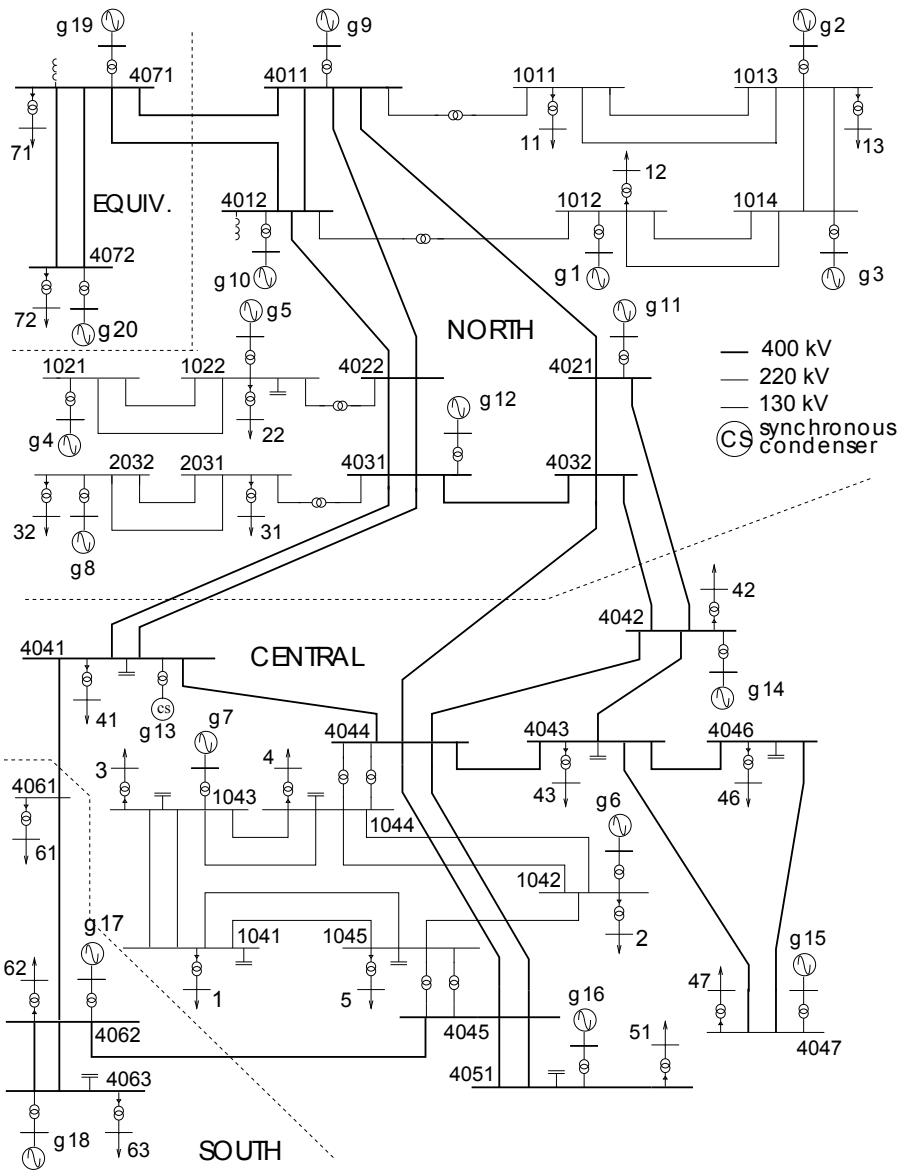

Figure 4. One-line diagram of the Nordic test system [17]

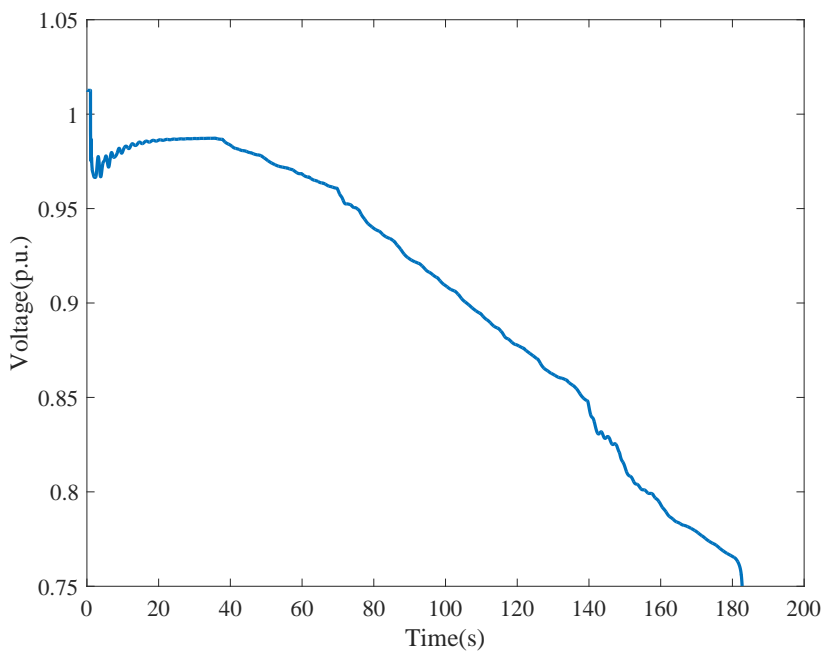

Figure 5. Voltage (in pu) at bus 1041 in Case A

$\left(S_{l d}<S_{\max m}\right)$. In fact, the load power decreases under the effect of the curtailment, while the maximum power increases under the effect of the resulting stabilization. The difference between both curves shows a growing stability margin.

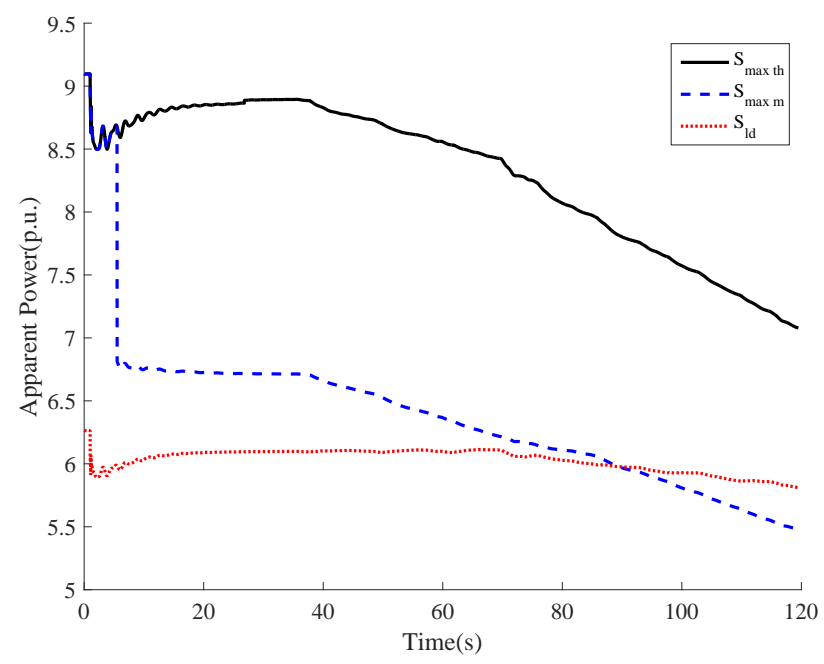

Figure 6. Maximum power (in pu) computed with TEM and compared to load power at bus 1041 in Case A

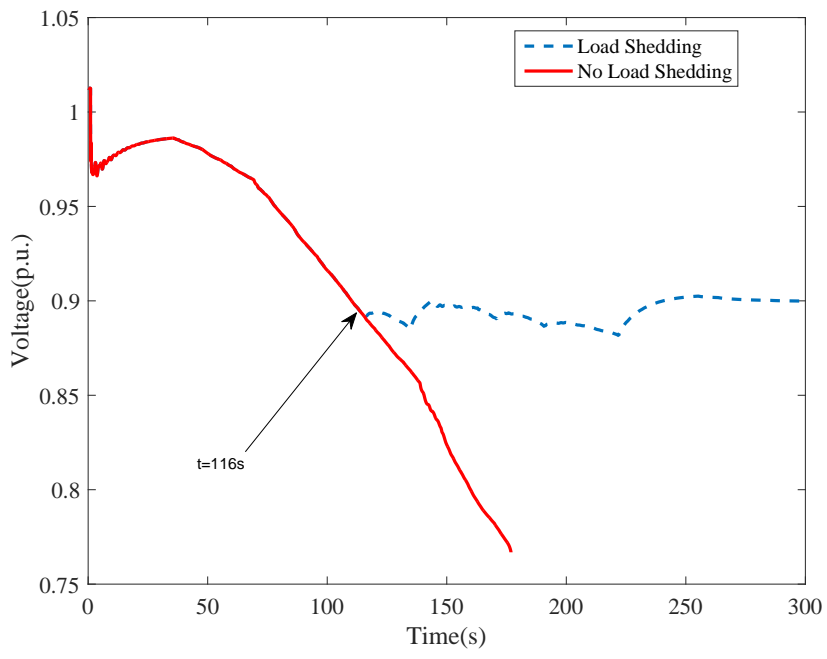

Figure 7. Voltage (in pu) at bus 1041 in Case B

\section{DISCUSSION}

Before commenting on possible synergies between the improved TEM and the sensitivity-based method, the latter is briefly recalled.

\section{A. The sensitivity-based method}

The sensitivity-based method for detection of voltage instability, in the general case involving multiple loads and multiple generators, was proposed in [10].

The sensitivities $S_{Q_{g} Q_{i}}$ of the total reactive power generation $Q_{g}$ to the various individual reactive power loads $Q_{i}$ are considered. Their abrupt change from large positive to large negative values at some buses is the signature of the fact that a combination of load active and reactive powers passes through a maximum. This provides a clear indication of a developing instability, together with a ranking of the affected buses.

These sensitivities are computed from an extended set of equilibrium equations fitted to the system states computed or 


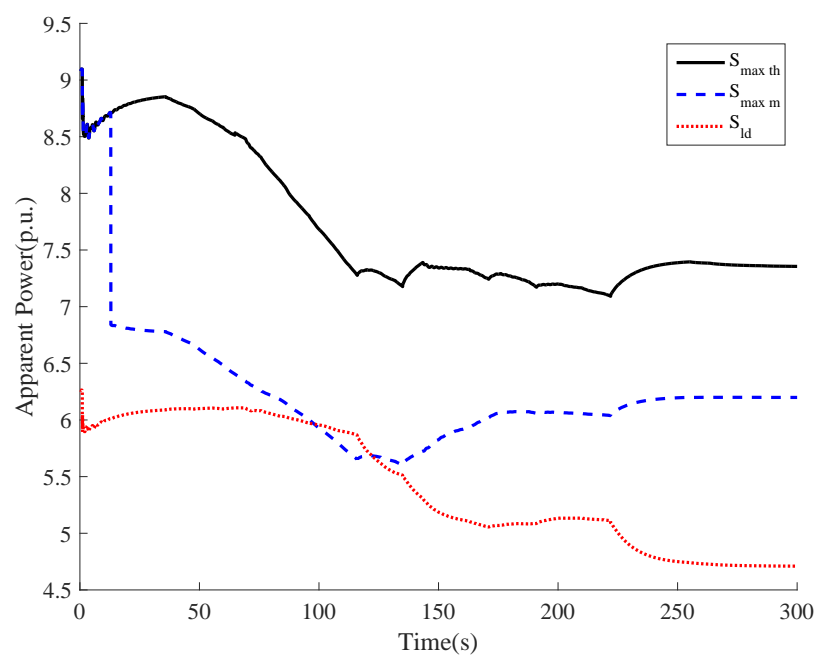

Figure 8. Maximum power (in pu) computed with TEM and compared to load power at bus 1041 in Case B

estimated from successive samples of available synchrophasor measurements. The status of each OEL is either transmitted or detected from the computation of the field current. The anticipation of the field current limitation was proposed in [16], which has inspired the improvement of the TEM presented in this paper.

Full observability of the system is assumed from the synchrophasor measurements. However, the case with only a limited number of them is dealt with in [18].

\section{B. Comparison between TEM and sensitivity-based indices}

For the sake of comparison, Fig. 9 presents the TEM and the sensitivity results relative to Case A, previously considered in Figs. 5 and 6. Both methods can identify the crossing in the stability boundary, and produce an early warning. The earliest warning is given by the change of sign of sensitivities, followed by the margin obtained from the improved TEM. At the time the sensitivities change sign, the margin given by the improved TEM has decreased to $1 \%$ of $S_{\max m}$.

\section{Synergy between TEM and sensitivity-based method}

The two approaches have different features regarding the information they provide:

- the TEM provides a distance to instability boundary, in the form of (active, reactive or apparent) power margins (as computed by this method);

- the sensitivities $S_{Q_{g} Q_{i}}$ (more precisely their change of sign from a large positive to a large negative value) shows accurately when this boundary is crossed;

- the limitations of the TEM, previously identified in $[11,13,14]$, are significantly removed by including the corrections proposed in this paper. Yet, the method can benefit from the complementary indication given by the sensitivities.

A possible synergy between both approaches is sketched in Fig. 10. The progressively decreasing security margin given

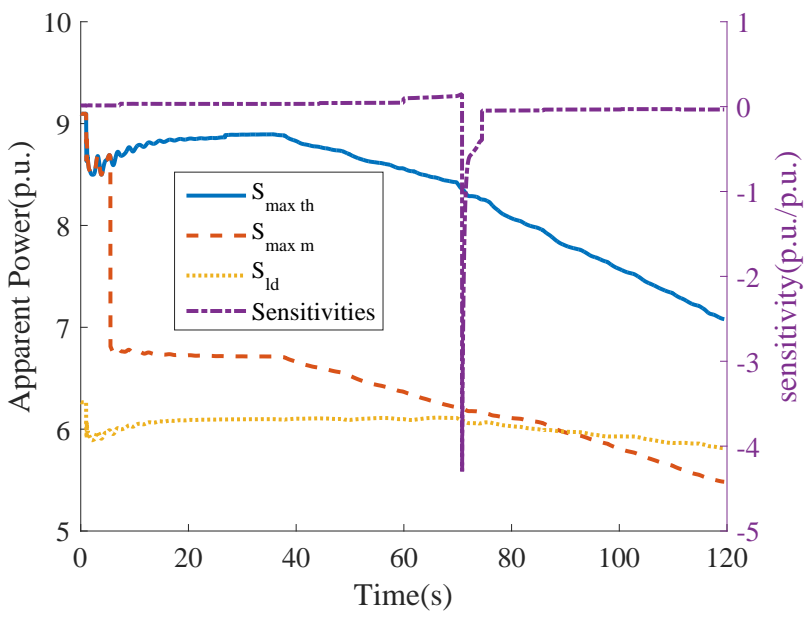

Figure 9. Maximum power (in pu) computed with the TEM, compared with the sensitivities; bus 1041 in Case A

by the TEM serves as a early warning of the events, while the sensitivities provide the definitive warning from which emergency actions can be taken by operators or by dedicated controllers.

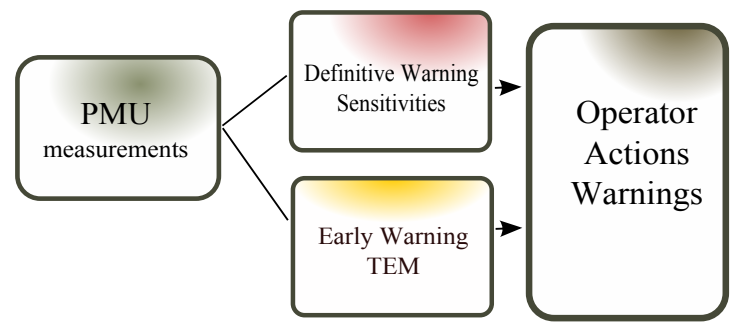

Figure 10. Synergy between Thévenin equivalent and sensitivitity-based methods

In the above context, the location and stability margin information provided by TEM needs to be further explored for its use in prosumption control and other counteractive actions. As regards sensitivities, a two-level scheme has been proposed in [22] combining a wide-area monitoring with local controllers aimed at maintaining transmission voltages at their values when the alarm has been received. It would be of interest to make this scheme further benefit from the TEM features.

\section{CONCLUSION}

An improved TEM that anticipates activation of synchronous generator's OEL was presented in this paper. The improvement offers more accurate computations of stability margins and early indication of impending voltage instability. This is illustrated through comparisons with previously proposed TEM in long-term voltage unstable and stabilized situations using the Nordic test system. The application of the TEM is thus enhanced and its anticipation capability can be used to prioritize and activate control actions and countermeasures. The limitations of the TEM, identified in $[11,13,14]$, are 
significantly removed by including the corrections proposed in this paper; it highlights the importance of considering the OELs and the importance of the Nordic system study cases in the development of voltage stability assessment methods.

The initial exploration of a possible synergy with the sensitivity-based method indicates that more information can be available for the operator and automatic control modules if both approaches are used in parallel. This synergy is worth being further explored in the following directions:

- a thorough comparison between the improved TEM and the sensitivity-based method, in particular in marginally stable or unstable cases;

- the combined use of both methods to scale the control actions, or include them in the framework detailed in [22].

\section{REFERENCES}

[1] A. Phadke and R. de Moraes, "The wide world of widearea measurement," Power and Energy Magazine, IEEE, vol. 6, no. 5, pp. 52-65, September 2008.

[2] V. Madani et al., "Challenging changing landscapes: Implementing synchrophasor technology in grid operations in the wecc region," Power and Energy Magazine, IEEE, vol. 13, no. 5, pp. 18-28, Sept 2015.

[3] H. Morais et al., "Sospo-sp: Secure operation of sustainable power systems simulation platform for real-time system state evaluation and control," IEEE Transactions on In Industrial Informatics, vol. 10, no. 4, pp. 23182329, Nov 2014.

[4] E. Dmitrova, M. Wittrock, H. Jóhannsson, and A. H. Nielsen, "Early prevention method for power system instability," Power Systems, IEEE Transactions on, vol. 30, no. 4, pp. 1784-1792, July 2015.

[5] H. Jóhannsson, A. H. Nielsen, and J. Østergaard, "Widearea assessment of aperiodic small signal rotor angle stability in real-time," IEEE Transactions on Power Systems, vol. 28, no. 4, pp. 4545-4557, 2013.

[6] J. T. G. Weckesser, H. Jóhannsson, J. Østergaard, and T. Van Cutsem, "Sensitivity based Assessment of Transient Voltage Sags caused by Rotor Swings," in PSCC14 - the 18th Power Systems Computation Conference, Wroclaw, Poland, Aug. 2014.

[7] S. Sommer and H. Jóhannsson, "Real-time thevenin impedance computation," 2013 IEEE PES Innovative Smart Grid Technologies Conference (ISGT), pp. 1-6, Feb. 2013.

[8] P. Kundur et al., "Definition and classification of power system stability ieee/cigre joint task force on stability terms and definitions," Power Systems, IEEE Transactions on, vol. 19, no. 3, pp. 1387-1401, Aug 2004.

[9] T. Van Cutsem, "Voltage instability: phenomena, countermeasures, and analysis methods," Proceedings of the IEEE, vol. 88, no. 2, pp. 208-227, 2000.

[10] M. Glavic and T. Van Cutsem, "Wide-area detection of voltage instability from synchronized phasor measurements. Part I: Principle," IEEE Transactions on Power Systems, vol. 24, no. 3, pp. 1408-1416, 2009.
[11] A. Perez, H. Jóhannsson, P. Vancraeyveld, and J. Østergaard, "Suitability of voltage stability study methods for real-time assessment," in Innovative Smart Grid Technologies Europe (ISGT EUROPE), 2013 4th IEEE/PES, Oct 2013, pp. 1-5.

[12] M. Glavic and T. Van Cutsem, "A short survey of methods for voltage instability detection," in Power and Energy Society General Meeting, 2011 IEEE, July 2011, pp. 1-8.

[13] A. Perez, H. Jóhannsson, and J. Østergaard, "Evaluation of enhancements to thévenin equivalent based methods for real-time voltage stability assessment," in Innovative Smart Grid Technologies Conference Europe (ISGTEurope), 2014 IEEE PES, Oct 2014, pp. 1-5.

[14] — "Wind farms generation limits and its impact in real-time voltage stability assessment," in PowerTech, 2015 IEEE Eindhoven, June 2015, pp. 1-5.

[15] —, "Evaluation of hvdc interconnection models for considering its impact in real-time voltage stability assessment," in IEEE MEPS poland, 2015, pp. 1-5.

[16] M. Glavic and T. Van Cutsem, "Wide-Area Detection of Voltage Instability From Synchronized Phasor Measurements. Part II: Simulation Results," IEEE Transactions on Power Systems, vol. 24, no. 3, pp. 1417-1425, Aug. 2009.

[17] T. Van Cutsem and L. Papangelis, "Description, modeling and simulation results of a test system for voltage stability analysis," Université de Liège, Internal report, Nov 2013.

[18] M. Glavic and T. Van Cutsem, "State reconstruction from a limited number of synchronized phasor measurements: Application to voltage instability detection," 2012 IEEE Power and Energy Society General Meeting, pp. 1-8, Jul. 2012.

[19] J. Lavenius, L. Vanfretti, and G. Taranto, "Performance assessment of pmu-based estimation methods of thevenin equivalents for real-time voltage stability monitoring," in Environment and Electrical Engineering (EEEIC), 2015 IEEE 15th International Conference on, June 2015, pp. 1977-1982.

[20] A. Perez, H. Jóhannsson, K. Martin, and J. Østergaard, 'Improved method for considering pmu's uncertainty and its effect on real-time stability assessment methods based on thévenin equivalent," in PowerTech, 2015 IEEE Eindhoven, June 2015, pp. 1-5.

[21] P. Aristidou, D. Fabozzi, and T. Van Cutsem, "Dynamic simulation of large-scale power systems using a parallel schur-complement-based decomposition method," Parallel and Distributed Systems, IEEE Transactions on, vol. 25, no. 10, pp. 2561-2570, Oct 2014.

[22] B. Otomega, M. Glavic, and T. Van Cutsem, "A two-level emergency control scheme against power system voltage instability," Control Engineering Practice, vol. 30, pp. 93 - 104, 2014. [Online]. Available: http://www.sciencedirect.com/science/article/ pii/S0967066113001895 